\title{
Población ocupada juvenil \\ en el mercado de trabajo rural uruguayo, década 2000*
}

\author{
The young working population in the rural uruguayan \\ labor market: year 2000
}

\section{Povoação Juventude ocupada no Mercado de Trabalho Rural Uruguaio década 2000}

\section{Juan Ignacio Romero Cabrera** - Uruguay}

Recibido el 28 de febrero de 2012, aceptado el 17 de mayo de 2012

\section{Resumen}

Objetivo: analizar las principales características y tendencias de las ocupaciones no agrarias en el territorio rural uruguayo, a partir de las características del empleo en el pasado lustro. Metodología: enfoque cuantitativo. Se pretendió debatir las herramientas conceptuales sobre las transformaciones productivas, reestructuración del proceso capitalista de producción y su impacto en los territorios rurales. Se utilizaron como fuentes de datos secundarios las Encuestas Continuas de Hogares de 2007 a 2010 y la Encuesta Nacional de Hogares Ampliados de 2006 del Instituto Nacional de Estadística. Resultados: los datos presentaron tendencias diferenciales en los procesos de emergencia y consolidación de ocupaciones no agrarias en diferentes territorios rurales, permitiendo interpretar este proceso social como heterogéneo. Conclusiones: la aplicación del enfoque conceptual planteado no tendría el mismo grado de validez para los territorios rurales del Uruguay, dada las diferentes dinámicas socioproductivas que generan una distribución heterogénea de las ocupaciones no agrarias en los mercados de trabajo regionales.

Palabras claves: juventud, empleo no agrario, ocupaciones no agrarias, territorio rural, desarrollo rural

* Este artículo es resultado del proyecto de investigación "Transformaciones socioproductivas en el territorio rural del litoral oeste: análisis del mercado de empleo desde la dimensión territorial y generacional", que forma parte de las actividades de investigación integral del autor en el Programa Ruralidad y Desarrollo Territorial de la Unidad de estudios regionales. Facultad de ciencias sociales de la Universidad de la República. Uruguay.

** Doctor en Sociología. Sociólogo. Docente e investigador de la Universidad de la República (Uruguay). Investigador Nivel I de la Agencia Nacional de Innovación e Investigación (Uruguay). juanromero69@gmail.com. 


\section{Abstract}

Objective: To analyze the main features and trends of non-agricultural occupations in rural Uruguay using employment characteristics from the past five years. Methodology: A quantitative approach was employed with the aim of discussing conceptual tools with respect to transformations in production, the restructuring of the capitalist production process and its impact on rural areas. The Continuous Household Surveys from 2007 to 2010 and the National Household Survey of 2006, produced by the Comprehensive National Institute of Statistics, were used as secondary data sources. Results: The data showed different trends in the processes of emergence and consolidation of non-agricultural occupations in different rural areas, which allowed this social process to be interpreted as heterogeneous. Conclusions: The implementation of the proposed conceptual approach would not have the same degree of validity for the rural areas of Uruguay given the different socio-productive dynamics that generate a heterogeneous distribution of non-agricultural occupations in the regional labor markets.

Keywords: youth, non-agricultural employment, non-agricultural occupations, rural area, rural development

\section{Resumo}

Objetivo: analisar as principais características e tendências das ocupações não agrárias no território rural uruguaio, a partir das características do emprego no passado lustro. Metodologia: enfoque quantitativo. Pretendeu se debater as ferramentas conceptuais sobre as transformações produtivas, reestruturação do processo capitalista de produção e seu impacto nos territórios rurais. Utilizaram se como fontes de dados secundários nas Enquetes Continuas de Lares de 2007 a 2010 e a Enquete Nacional de Lares Ampliados de 2006 do Instituto Nacional de Enquete. Resultados: Os dados apresentaram tendências diferenciais nos processo de emergência e consolidação de ocupações não agrárias em diferentes territórios rurais, permitindo interpretar este processo social como heterogêneo. Conclusões: A aplicação do enfoque conceptual apresentado não teria o mesmo grau de validez para os territórios rurais de Uruguai, proporcionadas as diferentes dinâmicas sócio - produtivas que geram uma distribuição heterogênea das ocupações não agrárias nos mercados de trabalhos regionais.

Palavras chaves: juventude emprego não agrário, ocupações não agrárias, territó144 rio rural, desenvolvimento rural.

Para citar este artículo:
Romero, J. (julio-diciembre, 2012). Población ocupada juvenil en el mercado de trabajo rural uruguayo, década 2000. Ánfora, 19 (33), 143-161. Universidad Autónoma de Manizales. ISSN 0121-6538. 


\section{Introducción}

En las últimas décadas la sociología rural inició un proceso de cambio sobre su forma de "mirar" al mundo rural; este proceso se inicia con fuertes críticas al modo productivista de los ambientalistas, lo que ha significado cuestionar la tradicional equivalencia entre desarrollo rural y desarrollo agrario. Este debate todavía continua abierto y muchas de sus "caras" no se profundizan lo suficiente. Para los fines de este estudio, interesa señalar algunos de los efectos que tuvieron estos cuestionamientos en la sociología rural.

La perspectiva central de la nueva ruralidad que ofrece estas posibilidades (no queda claro qué posibilidades ofrece la nueva ruralidad) tiene su énfasis en la cuestión territorial, cuyo análisis no deja de lado las dinámicas sociales y de producción agraria que se desarrollan en el territorio, aunque no hace de ellas el eje exclusivo del análisis.

Por lo tanto, la pertinencia de este enfoque territorial tendrá como uno de sus principales indicadores el tipo de ocupación existente: cuanto mayor sea la diversidad de ocupaciones existentes en un territorio mayor sería la pertinencia en la aplicación de los conceptos de nueva ruralidad y su abordaje territorial de los problemas del desarrollo. En tanto, si el nivel de ocupación no agrícola es muy bajo es necesario un análisis centrado en los procesos sociales agrarios para interpretar su situación presente y pensar en su desarrollo.

Entonces, el aumento del empleo no agrícola en los espacios rurales suscitaría nuevas interrelaciones entre lo urbano y lo rural, conformando también nuevas dinámicas territoriales. En definitiva el territorio rural incluye la agricultura y no viceversa.

Por este motivo, en este trabajo se intentará analizar las principales características de las ocupaciones en el territorio rural uruguayo para conocer el impacto a escala territorial de la pertinencia en el marco analítico del enfoque de la nueva ruralidad y la inclusión de la dimensión generacional. El mismo, es resultado del proyecto “'Transformaciones Socioproductivas en el Territorio Rural del Litoral Oeste: análisis del mercado de empleo desde la dimensión territorial y generacional", el cual forma parte de las actividades de investigación integral del autor en el Programa Ruralidad y Desarrollo Territorial de la Unidad de Estudios Regionales - Facultad de Ciencias Sociales.

\section{Breve contexto conceptual}

Terry Marsden (1993) es uno de los estudiosos europeos que se han ocupado de analizar las transformaciones socio-económicas globales y sus efectos sobre la agricultura y el espacio rural. Definió el concepto de reestructuración como un proceso de

Las cuales incorporan en sus diseños muestrales las localidades menores de 5000 habitantes. 
transformación global que puede ser observado más claramente en el sector industrial, caracterizado por la influencia creciente de las tecnologías de la informática y la microelectrónica. Sus impactos alcanzan también a las formas de organización productiva (nuevos procesos de producción) y, consecuentemente sobre las relaciones de trabajo (Marsden et al, 1993; Marsden, Lowe, Whatmore, 1990, Schneider, 2003).

En la perspectiva de Marsden, esta nueva fase se caracterizaría por la flexibilización de los procesos productivos, por la descentralización de las unidades de producción y por la informalización de las relaciones de trabajo, reduciéndose sustancialmente el papel del Estado y de las instituciones reguladoras.

En lo que respecta a la agricultura y al mundo rural, los efectos de la reestructuración económica, productiva e institucional pueden ser percibidos por medio de múltiples dimensiones. En primer lugar, se abren los mercados, se aceleran los intercambios comerciales y se intensifica la competitividad teniendo como base las poderosas cadenas agroalimentarias que monopolizan la producción y el comercio mayorista a escala global, restringiendo la participación en estas relaciones comerciales a grandes regiones productoras de los países (Reardon y Berdegué, 2003).

En segundo lugar, paralelamente al proceso continuo de profundización del progreso tecnológico (vía biotecnologías, ingeniería genética, etc.) se observa el surgimiento de iniciativas de los más variados matices, que cuestionan la sustentabilidad del modelo técnico dominante (Goodman y Watts, 1997). Dicho cuestionamiento apunta a las externalidades generadas por la intensificación del proceso de acumulación, lo que ha provocado serios desequilibrios sociales, económicos y en los últimos 30 se señalan los ambientales, colocando en riesgo las condiciones ecológicas para la reproducción de la vida humana.

El nuevo régimen de acumulación capitalista requiere de flexibilidad, ya sea para los procesos de producción como para las relaciones de trabajo que los componen, tendencia que se encuentra formando parte del movimiento de transnacionalización del capital lo que puede ser traducido como el control internacional capitalista de los locales de producción por un lado, y de los trabajadores geográficamente separados por otro. Esto aliado a la tendencia general de descentralización de la gestión estatal, provocó cambios en la comprensión y control (normativo) de los espacios nacionales los cuales son cada mas atravesados por los procesos productivos transnacionales.

La discusión del concepto de territorio tiene su retorno marcado por un período histórico caracterizado por la unión de la ciencia y la tecnología con la información, este nuevo medio geográfico posee manifestaciones puntuales, que están relacionados a procesos encadenados, mostrando su carácter universal (Santos, 1999; Schneider, 2005).

El retorno del territorio, como mencionó Milton Santos (1994) se encuentra relacionado con los cambios socio-espaciales e político-institucionales del capitalismo en su

Para citar este artículo:
Romero, J. (julio-diciembre, 2012). Población ocupada juvenil en el mercado de trabajo rural uruguayo, década 2000. Ánfora, 19 (33), 143-161. Universidad Autónoma de Manizales. ISSN 0121-6538. 
fase póst-fordista (Harvey, 1993). En este sentido, el territorio emerge como un proceso vinculado a la globalización, sobre todo porque la nueva dinámica económica y productiva depende de decisiones e iniciativas que son tomadas en función del territorio.

También emerge como una nueva unidad de referencia para la actuación del Estado y la regulación de las políticas públicas, para que este accionar fuese efectivo, las relaciones entre el Estado central y los organismos locales fue necesario construir una nueva unidad de referencia, que paso a ser el territorio y por consecuencia las acciones de intervención en el mismo se denominaron desarrollo territorial.

Un segundo elemento que puede ser atribuido como motivo para la emergencia del abordaje territorial, está basado en el cuestionamiento creciente de la dinámica sectorial de ramas de actividad económica, que pasó a desarrollarse más en una lógica de obtención de objetivos productivos que de escala. Por lo que el abordaje territorial promovió la superación del enfoque sectorial de las actividades económicas (agricultura, industria, comercio, servicios, etc.) y reemplazó la dicotomía espacial entre lo rural versus urbano o campo versus ciudad (si se plantea la dicotomía rura/urbano, mediado por versus queda más antagónico que dicotómico) En la perspectiva territorial, las dicotomías y los antagonismos son substituidos por la diversidad de acciones, estrategias y trayectorias que los actores (personas, empresas o instituciones) adoptan teniendo presente su reproducción social y económica.

En este sentido, el territorio emerge como un proceso vinculado a la globalización y conceptuado como una unidad espacial integrada por un tejido social con identidad particular, que tiene como sustento material una determinada base productiva de recursos naturales, articulada con otras formas de producción y de organización del trabajo, consumo e intercambio, coordinado por instituciones y formas de organización que actúan en el mismo.

Los cambios mencionados de la fase post-fordista, nos colocan en la necesidad de examinar el cuerpo conceptual que posibilitaba interpretar los procesos sociales de la sociedad rural latinoamericana, el cual comienza a ser cuestionado en sus supuestos, es decir, en la forma dicotómica de analizar la realidad social rural bajo categorías como: modernidad - tradicional; civilización - barbarie; rural - urbano. (modernidad/ tradicional)

Esta visión de la ruralidad resaltaba dos aspectos:

a) Establecía una coincidencia entre lo rural y lo agrícola.

b) Lo rural venía a ser una categoría residual frente a lo urbano (Gómez, 2002, p. 199).

Este debate no es nuevo (Rozman, 1930), pero desde la década del 80, los resultados de las investigaciones sociales instalaron la importancia de otros factores aparte de la lógica de la unidad familiar, tales como: composición y ciclo vital de la familia, presen- 
cia del mercado de trabajo no agrícola y diversas características del contexto regional y local, inclusive políticas en la conformación y evolución de los empleos múltiples (Múltiple Job Household). Estos por definición, se refieren específicamente a las actividades remuneradas con una base contractual convencional.

Al final de esta década la ampliación del concepto se dirigió a incluir ocupaciones no necesariamente remuneradas en dinero, lo que llevó a la investigación de Arkleton Trust a la adopción del término pluriactividad, lo cual puede incluir:

a) Empleo en otros establecimientos agrícolas de forma asalariada;

b) Actividades "para agrícolas" como alimentos y bebidas procesadas;

c) Actividades no agrícolas en el establecimiento como turismo y hotelería;

d) Actividades externas no agrícolas como emplearse de forma asalariada en otra rama de actividad (Fuller, 1990, p. 367; Kageyama, 2000).

Lo que estaría importando sería la existencia del mercado de trabajo con necesidades que pueden ser satisfechas por los miembros de las familias rurales pluriactivas, las cuales no dependerían de la delimitación urbana - rural y si de las características económicas, sociales y culturales de las economías locales y regionales. Ante ello, los aportes de los estudios sobre la pluriactividad incorporaron la conceptualización de que no todas las actividades económicas en el medio rural serían de perfil agrícola y que existirían diferentes formas de remuneración.

Ahora, también se observa otra mirada teórica, en la cual el énfasis estaría sobre la distribución espacial en un territorio determinado, es decir, lo rural no es definido por la oposición y si por la relación con las ciudades (Abramovay, 2000).

Entonces, lo que estaría siendo planteado sería que la unidad de análisis sean las economías regionales y específicamente aquellas donde las personas viven en áreas poblacionales menos densas que en el resto del país. En otras palabras, el concepto de desarrollo rural se constituye con base en lo espacial y multisectorial desde el punto de vista productivo del cual la agricultura forma parte.

Para el caso uruguayo, los procesos antes mencionados se consolidan y profundizan en los pasados 30 años pero tienen sus inicios a finales de la década de 1950 con la crisis del modelo de sustitución de importaciones y el inicio de la retirada gradual del Estado de bienestar. Lo que significó la baja de aranceles a la importación de productos agropecuarios, la quita de apoyos y subsidios; la promoción de rubros no tradicionales de exportación lo que se tradujo en un crecimiento extraordinario de la producción y exportación de ciertos rubros como ser: arroz, citrus, lácteos, cebada cervecera y recientemente la soja y la forestación configurando cadenas agroindustriales integradas al mercado global; procesos de cambio tecnológico procurando disminuir la fuerza 148 de trabajo y aumentar la productividad; cambios en la estructura agraria tendiendo 
a una mayor concentración de la tenencia de la tierra en grandes superficies y en los últimos 10 años a la extranjerización de dicha tenencia; disminución de la población rural siendo uno de los países de América Latina con mayores índices de urbanización y en los últimos 5 años crecimiento de las exportaciones y reinversión en el sector. Estos procesos han convergido en una mayor asalarización de la mano de obra rural uruguaya (aproximadamente 6 de cada 10 trabajadores rurales), encontrándose dentro de los países con mayor índice en tal sentido; procesos semejantes a los acontecidos en la pradera pampeana argentina (Piñeiro, 2000).

Por último, el profesor Abramovay, agrega que existirían tres aspectos básicos en la literatura internacional en la línea del pensamiento mencionado sobre el medio rural: a) la relación con la naturaleza; b) la importancia de las áreas no densamente pobladas y c) la dependencia del sistema urbano. En definitiva, la forma dicotómica de analizar la realidad social rural ha entrado en crisis, una de las "llaves" para ello ha sido el observar el proceso de cambio ocurrido con la categoría ocupación, la cual hoy en día se presenta como no ligada al espacio geográfico necesariamente y como desafío para las herramientas teóricas.

\section{Metodología}

El análisis de la información tomó como principal fuente de datos secundarios las Encuestas Continuas de Hogares (ECH) de 2007 a $2010^{1}$ y la Encuesta Nacional de Hogares Ampliados (ENHA) de 2006 del Instituto Nacional de Estadística INE. La decisión de tomar el período 2006 al 2010 implicó considerar un período de crecimiento económico dinámico e intenso comparado con los 20 años anteriores; esto por un lado. Por otro, porque toma en cuenta el período de gubernamental del primer gobierno de izquierda en el Uruguay y se procura analizar las posibles características de dicho crecimiento económico en un contexto político diferente al histórico.

Optar por el análisis de datos de origen secundario como las señaladas arriba, permiten observar las tendencias a nivel macro, por territorios y detectar las principales tendencias estructurales especialmente desde el enfoque generacional que ha sido escasamente explorado.

Debido a la (en adelante INE) extensa cantidad de categorías que identifican el tipo de empleo que desarrollan las personas de los territorios rurales, se recodificaron las mismas en siete (7) categorías de ocupación que abarcan las tres (3) ramas de actividad. Dichas categorías son: 1- Miembros Poder Ejecutivo (PE) PE, dirigentes empresas, ejecutivos; 2- Profesionales, científicos, profesores secundaria, maestros titulados; 3- empleados oficina, técnicos medios, escritores, artistas; 4- trabajadores servicios, comercios; 5- trabajadores y agricultores calificados explotaciones primarias; 6- trabajadores de industrias, minería, operarios montajes y maquinaria y 7 - trabajadores no calificados.

Para citar este artículo:
Romero, J. (julio-diciembre, 2012). Población ocupada juvenil en el mercado de trabajo rural uruguayo, década 2000. Ánfora, 19 (33), 143-161. Universidad Autónoma de Manizales. ISSN 0121-6538. 
Con el procesador estadístico Statistical Package for Social Sciences SPSS se consiguió reconstruir dichas bases tomando como universo de análisis los territorios rurales, aislando de esta manera los centros urbanos mayores de 10000 habitantes para posteriormente centrar el análisis en las características de la Población Económicamente Activa PEA de acuerdo al problema planteado.

\section{Resultados}

\section{La dimensión generacional en el análisis del problema}

Para una aproximación al modo en que fueron abordados los problemas sociales de los jóvenes uruguayos, se analizarán los temas que emergieron en el análisis de los diversos autores que se ocuparon de la problemática desde el origen de la sociología como ciencia social en el país a lo largo de estos 30 años.

En el período de democratización, de 1985 a 1990, algunos trabajos vuelven a problematizar la actuación de los diversos movimientos sociales de jóvenes de fines de los 60 (Rodríguez, 1985; Terra, 1985). Sin embargo, no se puede afirmar que en este período los jóvenes sean considerados esencialmente como militantes, ya que estos análisis buscan sobre todo saldar la comprensión histórica de los acontecimientos del período pre-dictatorial. Son también considerados como trabajadores o futuros trabajadores, siendo muchos de ellos desempleados, como estudiantes, menores peligrosos y madres adolescentes (Cepal, 1988, 1989; Filgueira, Rama, 1991; Diez de Medina, 1989; Rama, 1989; Solari, 1989). Los jóvenes son, entonces, un sector social que enfrenta diversos problemas -la violencia, la educación, el trabajo, la conformación de una familia, la sexualidad-, en una comprensión que abandona el problema del estancamiento social y la falta de oportunidades de ascenso para superar el de la "exclusión". Esta inflexión hace que muchos trabajos sociológicos dejen de centrarse en el problema de la movilidad social y en el análisis global de la sociedad, para pasar a un punto de vista fragmentado, que analiza específicamente determinados sectores sociales (especialmente los excluidos socialmente) y las problemáticas que sufren (Lovesio, y Viscardi, 2003).

En este contexto de análisis teórico, se pretende aportar a la conceptualización de la categoría juventud rural y se entiende que las normas, valores, prácticas relacionales y en general, la visión de mundo de los jóvenes, parte de los referentes culturales particulares del grupo social donde éstos viven el proceso de socialización. El ser joven se da en espacios institucionales centrales como la familia, la escuela, el colegio y o lugar de trabajo, y en núcleos más informales pero muy influyentes como el grupo de amistades. A manera de múltiples espejos, la visión que el joven construye de sí mismo tiene relación con la forma en que mira la sociedad, y ésta, a la vez, se refleja en sus jóvenes con toda su fuerza contradictoria.

Para citar este artículo:
Romero, J. (julio-diciembre, 2012). Población ocupada juvenil en el mercado de trabajo rural uruguayo, década 2000. Ánfora, 19 (33), 143-161. Universidad Autónoma de Manizales. ISSN 0121-6538. 
En este sentido y con el apoyo de la conceptualización de Durston, se entiende que se deberían tomar tres procesos distintos y simultáneos que influyen unos a otros en la conformación del joven: a) el ciclo de vida de la persona; b) la evolución cíclica del hogar en que la persona vive; y c) las relaciones intergeneracionales e intrageneracionales, que surgen en gran medida de la interacción entre el ciclo de vida del hijo y el de la evolución de su hogar de socialización. Estas dimensiones conceptuales son presentadas a manera de esbozo teórico y que permitirían orientar la interpretación de los resultados empíricos, en especial lo relacionado al primer punto. Por otra parte, serían dimensiones analíticas a ser consideradas en lo que respecta a la transferencia de la propiedad de la tierra y la conformación de la nueva generación de productores impactados por el proceso de modernización agraria impulsado en la década del 70 (Romero, 2002).

Así, se ha señalado al conflicto intergeneracional como una de las causas de la invisibilización del aporte de la población joven al funcionamiento de la sociedad. En este orden de ideas, la subordinación del joven se relaciona al esquema patriarcal-autoritario en el cual el ejercicio del poder, por parte de las generaciones adultas, invisibiliza sus aportes y su potencial. Si bien el sistema patriarcal es un factor que incide directamente en la invisibilización de los jóvenes, ésta se revela como multicausal. La desigualdad estructural de las sociedades y la inequidad en la distribución de la riqueza social crea condiciones para la exclusión sistemática de sectores sociales que se realiza por uno u otro medio (IICA, 2000).

En definitiva, el objetivo prioritario del jefe de hogar/unidad productiva joven es el de la subsistencia/consumo; el de mediana edad se centra en la acumulación/capitalización y finalmente, el jefe mayor da prioridad al objetivo de maximizar su prestigio, sobre la base de una combinación de riqueza, poder, generosidad y servicio. Mientras que entre aquellos jóvenes que no poseen tierra, la presión intergeneracional es el de poder acceder a la misma y conformar su hogar o de aumentar sus activos educativos (en especial las mujeres) y emigrar a los espacios urbanos.

Ante esto, la etapa de la juventud es una etapa de especial tensión intergeneracional, en la que los intereses del jefe del hogar/unidad productiva (mayor de 30 años) presentan la posibilidad de iniciar un ciclo de posible acumulación y alejamiento de la pobreza al contar con la fuerza de trabajo de sus hijos mayores, nueras y yernos, coincide en el tiempo con el de máximo interés de los hijos en concretar y adelantar la ruptura de esa relación de dependencia y control. A lo que se suma el interés de los jóvenes por el cambio cultural y por las nuevas posibilidades de poder económico independiente que abren la educación y el trabajo asalariado.

Estas situaciones de conflicto presentan en la tierra uno de sus principales activos por los cuales la generación nueva con la anterior confrontan sus intereses, pero también, denota el tipo de desarrollo en el medio rural que posibilite la sustentabilidad del mismo. Es decir, muchos jóvenes se encuentran dispuestos a desarrollar sus estrategias 
de vida en el medio rural y no pueden formar su hogar y por lo tanto se les vuelve imposible materializar tales estrategias. En mucho de los casos, por la escasa renovación generacional en la propiedad de la tierra; por la falta de acceso a la misma vía líneas de créditos y políticas destinadas a promover productivamente al joven rural y también por la emigración juvenil al medio urbano, en especial de las mujeres.

Ahora, en la última década los estudios realizados en el continente latinoamericano han demostrado que estos jóvenes rurales incorporaron en sus estrategias de vida la vinculación al mercado de trabajo no agrario, el cual se ha presentado como una alternativa viable de desarrollo en el territorio rural pero por otra parte, un recurso humano capacitado, flexible y disponible para las estrategias empresariales no agrarias por un lado y las unidades de producción familiar por otro, situaciones que se observan en aquellos territorios que han consolidado procesos de modernización agraria e integración al mercado global o condiciones socioproductivas con escasa incorporación al proceso mencionado pero que han generado dinámicas de exclusión a los medios de producción.

Situación esta que evidencia mayor dinamismo de los rubros productivos no agrarios en los territorios rurales, impactando también en especial en los jóvenes lo que conlleva a repensar su conceptualización como categoría analítica dado que no exclusivamente se construye desde las relaciones sociales originadas en las actividades agrarias.

\section{Principales tendencias ocupacionales de los jóvenes rurales uruguayos}

El agro uruguayo ha sufrido en las últimas tres décadas una profunda transformación productiva a raíz de la introducción de nuevos rubros de producción y de una creciente integración agroindustrial, lo que derivó en una acelerada incorporación de cambios tecnológicos. Sin embargo, este fenómeno presenta la característica particular de haberse desarrollado fuera del sector tradicional de producción de carne y lana, el cual ha mantenido esencialmente las mismas formas de producción desde mediados de siglo, lo que ha generado, salvo algunas variaciones estacionales, su estancamiento de largo plazo. (Riella, Romero y Tubío, 1999).

Estas dos dinámicas diferenciadas dentro del sector ${ }^{1}$ han producido impactos territoriales muy heterogéneos dando lugar a una nueva regionalización de las áreas rurales. Sin embargo, la sociología rural no ha prestado mayor atención en estos procesos y a sus efectos sobre el territorio. Son muy escasos los estudios que se han preocupado por esta temática, y los que se han realizado han tenido un énfasis sectorial, por lo que no

1 El doble movimiento ha sido caracterizada como una situación de estancamiento dinámico, donde los sectores agroindustriales aportan el dinamismo al sector mientras la ganadería de carne y lana mantiene su producción global estancada. 
alcanzan a dar cuenta en forma exhaustiva de los efectos territoriales y el impacto que han tenido las actividades no- agrarias en el espacio rural de estas regiones del país.

Por otra parte, escasos son los estudios acerca de cómo estos cambios han generado o no dinámicas laborales diferenciales en los grupos etarios que integran el mercado laboral como un todo, en especial los jóvenes planteados como foco de análisis.

Un indicador de los cambios mencionados en el espacio social rural uruguayo, serían las transformaciones de los mercados de empleo y su impacto en los diferentes grupos de edades. Las características de alguno de estos mercados muestran una rearticulación de las interrelaciones entre la sociedad rural y la sociedad urbana, que puede hacer pertinente en algunos casos el uso del enfoque de la nueva ruralidad para comprender sus procesos sociales.

En el Uruguay la definición de ser joven es de tipo normativa, es decir, el Estado uruguayo determina entre que edades biológicas se entiende por ser joven. En este caso, comprende las edades entre los 14 y 29 años de acuerdo con lo establecido por la primera Encuesta Nacional de la Juventud al considerar el proceso de integración de los jóvenes a la sociedad, así como por la baja tasa de natalidad, llevada adelante por la Oficina CEPAL en Montevideo conjuntamente con la Dirección General de Estadísticas y Censos (DGEC) del Estado uruguayo instituto estadístico predecesor al actual INE.

Para lograr una aproximación a esta realidad laboral de los jóvenes, se analizará, en primera instancia, la evolución de las ocupaciones que llevan adelantes de acuerdo con la rama de actividad en dos momentos del tiempo: a principios de la década 2000 con la medición realizada por el Ministerio de Ganadería, Agricultura y Pesca (en adelante MGAP) en la Encuesta de Hogares Rurales ${ }^{1}$, y un segundo momento, a mediados de la década en el año 2006 analizando la información generado por la Encuesta Continua de Hogares (ECH en adelante) del INE y las sucesivas ECH hasta el 2010 para, de esta manera, delinear tendencias al respecto.

Posteriormente, se analizará la estructura ocupacional de las actividades que desarrollan los jóvenes en las distintas regiones rurales para apreciar en qué grado se han desarrollado los empleos no agrario y agrarios. Luego se analizará las ramas de actividad de los empleadores de los jóvenes rurales, para luego observar cómo se ha desarrollado la dinámica del mercado laboral y de qué manera se han insertado los mismos en los últimos años.

1 Encuesta de Hogares Rurales 2000. MGAP-OPYPA. Dicha encuesta tiene una definición de población rural amplia abarcando a los hogares de las localidades con menos de 5.000 habitantes es decir, 25\% de la población total que no esta integrada a la Encuesta Continua de Hogares (ECH) y sobre la cual se conocía muy poco ya que solo se contaba con los censos nacionales para el análisis de esta población, lo cuales ocurren cada 10 años. (Fuente: elaboración propia). 
A continuación se presenta entonces la evolución de las ocupaciones no agrarias y agrarios de los jóvenes rurales uruguayos en la presente década.

\section{Tabla 1 - Población ocupada joven territorios rurales según rama de actividad $2006-2010$}

\begin{tabular}{|l|c|c|c|c|c|c|c|}
\hline Rama de Actividad & $\begin{array}{c}\mathbf{2 0 0 0} \\
\mathbf{\%}\end{array}$ & $\begin{array}{c}\mathbf{2 0 0 6} \\
\mathbf{\%}\end{array}$ & $\begin{array}{c}\mathbf{2 0 0 7} \\
\mathbf{\%}\end{array}$ & $\begin{array}{c}\mathbf{2 0 0 8} \\
\mathbf{\%}\end{array}$ & $\begin{array}{c}\mathbf{2 0 0 9} \\
\mathbf{\%}\end{array}$ & $\begin{array}{c}\mathbf{2 0 1 0} \\
\mathbf{\%}\end{array}$ & Promedio del Período \\
\hline Primario & 58,4 & 47,0 & 46,0 & 23,0 & 55,0 & 46,0 & $\mathbf{4 5 , 9 \%}$ \\
\hline Secundario & 14,9 & 19,0 & 18,0 & 25,0 & 13,0 & 18,0 & $\mathbf{1 8 , 6 \%}$ \\
\hline Terciario & 26,6 & 33,0 & 36,0 & 52,0 & 32,0 & 36,0 & $\mathbf{3 7 , 8 \%}$ \\
\hline Total & 100,0 & 100,0 & 100,0 & 100,0 & 100,0 & 100,0 & \\
\hline
\end{tabular}

A partir de la década del 2000, con el innovador estudio llevado adelante por el MGAP, se comenzó a tener conocimiento sobre población especialmente de la sociedad rural que no era alcanzada por la ECH, lo que permitió ampliar la capacidad de análisis sobre la dinámicas sociales que estaban aconteciendo en dichos espacios sociales. Lo que se consolida en 2006 con la ENAH y posteriormente incluyendo a dichas localidades en la ECH, es por ello que la información que se presenta tiene como base el año 2000 y luego salta hacia el 2006 en adelante.

Según lo anterior, se observa que los jóvenes rurales ocupados desarrollan sus actividades mayoritariamente en la rama de actividad primaria. Le sigue en orden de importancia las ocupaciones desarrolladas en la rama de actividad terciaria y luego la secundaria o industrial, las cuales estarían formando parte del proceso de configuración espacial multifuncional del territorio rural uruguayo que presentará aspectos diferenciales en este sentido (Grammont, 2004).

Lo que se aprecia como tendencia es un constante crecimiento de las ocupaciones en la rama terciaria, de forma similar pero con un quiebre en dicho crecimiento en 2009 que la rama secundaria, pero reiniciando el mismo a partir de 2010; y una rama primaria que, aunque se presenta mayoritaria en las ocupaciones de los jóvenes rurales, presenta una tendencia a decrecer con momentos de crecimiento; es decir, un comportamiento inestable en dichas ocupaciones.

En definitiva, las ocupaciones de los jóvenes rurales vienen creciendo en la rama terciaria o de servicios, mientras que en la rama primaria (ocupaciones agrarias) presenta inestabilidad con momentos de crecimiento y decrecimiento; sin embargo, si se compara el inicio con el final de la década, la disminución ha sido de un $12,0 \%$ aproximadamente.

En la Tabla 2 el análisis se enfoca en las principales categorías ocupacionales que los 154 jóvenes rurales desarrollan en dicho mercado laboral en la pasada década.

\begin{tabular}{l|l} 
Para & Romero, J. (julio-diciembre, 2012). Población ocupada juvenil en el mercado de trabajo rural
\end{tabular} 
Tabla 2 - Principales categorías de ocupaciones de los jóvenes en territorios rurales $2006-2010$

\begin{tabular}{|l|c|c|c|c|c|c|}
\hline \multicolumn{1}{|c|}{ Principales Ocupaciones } & $\begin{array}{c}\mathbf{\%} \\
\mathbf{2 0 0 6}\end{array}$ & $\begin{array}{c}\mathbf{2 0 0 7} \\
\mathbf{2 0 0 0 8}\end{array}$ & $\begin{array}{c}\mathbf{2 0 0 9} \\
\mathbf{2}\end{array}$ & $\begin{array}{c}\mathbf{2 0 1 0} \\
\text { ejecutivos }\end{array}$ & $\begin{array}{c}\text { Promedio del } \\
\text { Período }\end{array}$ \\
\hline $\begin{array}{l}\text { Profesionales, científicos, profesores } \\
\text { secundaria, maestros titulados }\end{array}$ & 2,7 & 3,3 & 4,2 & 2,1 & 3,4 & $\mathbf{3 , 1 4 \%}$ \\
\hline $\begin{array}{l}\text { empleados oficina, técnicos medios, } \\
\text { escritores, artistas }\end{array}$ & 5,7 & 7,3 & 14,2 & 5,4 & 8,3 & $\mathbf{8 , 1 8 \%}$ \\
\hline trabajadores servicios, comercios & 10,7 & 12,7 & 18,7 & 12,7 & 13,4 & $\mathbf{1 3 , 6 4 \%}$ \\
\hline $\begin{array}{l}\text { trabajadores y agricultores } \\
\text { calificados explotaciones primarias }\end{array}$ & 16,3 & 11,4 & 4,9 & 15,0 & 12,8 & $\mathbf{1 2 , 0 8 \%}$ \\
\hline $\begin{array}{l}\text { trabajadores industrias, minería, } \\
\text { operarios montajes y maquinaria }\end{array}$ & 17,1 & 17,8 & 20,2 & 13,9 & 16,8 & $\mathbf{1 7 , 1 6 \%}$ \\
\hline trabajadores no calificados & 45,4 & 45,0 & 34,5 & 48,9 & 42,6 & $\mathbf{4 3 , 2 8 \%}$ \\
\hline Total & 100,0 & 100,0 & 100,0 & 100,0 & 100,0 & \\
\hline
\end{tabular}

Fuente: elaboración propia.

En este caso, la información hace referencia a los últimos 5 años. Como se aprecia, la categoría ocupacional mayoritaria que desarrollan los jóvenes es la de trabajador no calificado. La misma advierte una tendencia a la baja, de forma suavizada. Le sigue en orden de importancia, las ocupaciones en la industria, minería, manejo de maquinaria y como operarios en montajes industriales en la cual los jóvenes tienen una importancia porcentual y estratégica se ha mantenido estable. En un tercer orden, las ocupaciones en la tercer rama especialmente en el rubro comercial y de servicios la cual presenta una tendencia de crecimiento. En este sentido, pero con un peso mucho menor, se presentan las ocupaciones de mayor calificación en dicha rama como empleados de oficina, técnicos medios, ha presentado un importante crecimiento en este lustro. Y por último, las categorías ocupacionales de mayor calificación los jóvenes han tenido una participación estable.

En resumen, los jóvenes desarrollan ocupaciones mayoritariamente poco calificadas aunque describen una tendencia levemente decreciente; de la misma manera las ocupaciones en actividades agrarias presentan dicha tendencia, mientras que el aumento es provocado en ocupaciones terciarias como en los servicios, comercios, empleados de oficina, técnicos medios, lo que estaría indicando que la mayor capacitación formal de los mismos sumado a la demanda de tales oficios encuentra en los jóvenes el agente 
a desarrollar tales tareas, mientras que se mantiene estable su participación en las ocupaciones de mayor calificación.

En esta Tabla, la información da cuenta de las empresas por rama de actividad que emplean a los jóvenes en las diferentes ocupaciones que desarrollan sean agrarias o no agrarias.

Tabla 3 - Ramas de actividad de las empresas en las que se ocupan los jóvenes de los territorios rurales 2006 -2010

\begin{tabular}{|c|c|c|c|c|c|c|}
\hline $\begin{array}{l}\text { Ramas de } \\
\text { Actividad }\end{array}$ & $\begin{array}{c}\% \\
2006\end{array}$ & $\begin{array}{c}\% \\
2007\end{array}$ & $\begin{array}{c}\% \\
2008\end{array}$ & $\begin{array}{c}\% \\
2009\end{array}$ & $\begin{array}{c}\% \\
2010\end{array}$ & $\begin{array}{c}\text { Promedio del } \\
\text { Período }\end{array}$ \\
\hline rama primaria & 27,1 & 47,0 & 19,0 & 54,2 & 46,0 & $38,66 \%$ \\
\hline rama secundaria & 7,1 & 16,0 & 21,0 & 11,2 & 18,0 & $14,66 \%$ \\
\hline rama terciaria & 65,7 & 37,0 & 60,0 & 34,6 & 36,0 & $46,66 \%$ \\
\hline Total & 100,0 & 100,0 & 100,0 & 100,0 & 100,0 & \\
\hline
\end{tabular}

Fuente: elaboración propia.

Se observa que en la rama primaria (agrarias), las empresas tienden a aumentar su participación en el mercado laboral, de igual manera las de la rama secundaria (industria) y de forma decreciente las empresas de la rama terciaria (servicios).

Cuando se compara con las ocupaciones que desarrollan los jóvenes por rama, se observa que en el caso de las actividades agrarias son más los jóvenes que las empresas. En el caso de las actividades industriales es equitativa dicha distribución mientras que en la rama de los servicios son más las unidades productivas que los jóvenes ocupados en términos relativos, lo que estaría indicando dinámicas diferenciales en el mercado de trabajo rural juvenil.

Se estaría ante un escenario hipotético de sectores y territorios demandando mano de obra juvenil como sería el caso de las actividades primarias: en la industria, un escenario de estabilidad y en los servicios, una tendencia inestable que presenta en promedio mayor cantidad de unidades productivas con menores necesidades de demanda de mano de obra, que podría señalar la presencia y aumento en determinados momentos del ciclo económico de empresas unipersonales o familiares.

Las empresas agrarias se encuentran en un ciclo dinámico en el que se demande mano de obra juvenil. Más estable es la situación de las empresas industriales mientras 
que en los servicios la realidad es mas heterogénea dado que existiría una base amplia de PYMES con sustento de trabajo familiar y empresas que sí demandan empleo juvenil para ocupaciones de calificación media.

\section{Tabla 4 - Condiciones de empleo entre jóvenes y adultos de los territorios rurales $2006-2010$}

\begin{tabular}{|c|c|c|c|c|c|c|c|c|c|c|c|c|}
\hline \multirow{2}{*}{$\begin{array}{l}\text { Aproxi. } \\
\text { Calidad } \\
\text { del } \\
\text { Empleo }\end{array}$} & \multicolumn{2}{|c|}{2006} & \multicolumn{2}{|c|}{2007} & \multicolumn{2}{|c|}{2008} & \multicolumn{2}{|c|}{2009} & \multicolumn{2}{|c|}{2010} & \multirow{2}{*}{$\begin{array}{c}\begin{array}{c}\text { Promedio } \\
\text { del }\end{array} \\
\text { Período } \\
\text { Jóvenes }\end{array}$} & \multirow{2}{*}{$\begin{array}{c}\begin{array}{c}\text { Promedio } \\
\text { del }\end{array} \\
\text { Período } \\
\text { Adultos }\end{array}$} \\
\hline & Jóv. & Adult & Jóv. & Adult & Jóv. & Adult & Jóv. & Adult & Jóv. & Adult & & \\
\hline $\begin{array}{c}\% \text { de } \\
\text { Empleos } \\
\text { Formales }\end{array}$ & $49 \%$ & $46 \%$ & $52 \%$ & $49 \%$ & $58 \%$ & $51 \%$ & $48 \%$ & $49 \%$ & $55 \%$ & $38 \%$ & $52,4 \%$ & $46,6 \%$ \\
\hline Ingresos $^{1}$ & $\begin{array}{l}61 \% \\
\text { hasta } \\
1 \mathrm{smn}\end{array}$ & $\begin{array}{l}62 \% \\
\text { hasta } \\
1 \mathrm{smn}\end{array}$ & $\begin{array}{l}47 \% \\
\text { hasta } \\
1 \mathrm{smn}\end{array}$ & $\begin{array}{l}54 \% \\
\text { hasta } \\
1 \mathrm{smn}\end{array}$ & $\begin{array}{l}51 \% \\
\text { hasta } \\
1 \mathrm{smn}\end{array}$ & $\begin{array}{l}57 \% \\
\text { hasta } \\
1 \mathrm{smn}\end{array}$ & $\begin{array}{l}56 \% \\
\text { hasta } \\
1 \mathrm{smn}\end{array}$ & $\begin{array}{l}56 \% \\
\text { hasta } \\
1 \mathrm{smn}\end{array}$ & $\begin{array}{l}51 \% \\
\text { hasta } \\
1 \mathrm{smn}\end{array}$ & $\begin{array}{l}56 \% \\
\text { hasta } \\
1 \mathrm{smn}\end{array}$ & $\begin{array}{c}58 \% \\
\text { hasta } 1 \\
\text { smn }\end{array}$ & $\begin{array}{c}62 \% \\
\text { hasta } 1 \\
\text { smn }\end{array}$ \\
\hline $\begin{array}{c}\text { Horas } \\
\text { trabaj. } x \\
\text { semana }\end{array}$ & $\begin{array}{l}42 \% \\
48 \\
\text { hrs }\end{array}$ & $\begin{array}{l}46 \% \\
48 \\
\text { hrs }\end{array}$ & $\begin{array}{l}54 \% \\
48 \\
\text { hrs }\end{array}$ & $\begin{array}{l}51 \% \\
48 \\
\text { hrs }\end{array}$ & $\begin{array}{l}55 \% \\
48 \\
\text { hrs }\end{array}$ & $\begin{array}{l}52 \% \\
48 \\
\text { hrs }\end{array}$ & $\begin{array}{l}56 \% \\
48 \mathrm{hrs}\end{array}$ & $\begin{array}{l}55 \% \\
48 \mathrm{hrs}\end{array}$ & $\begin{array}{l}58 \% \\
48 \\
\text { hrs }\end{array}$ & $\begin{array}{l}53 \% \\
48 \\
\text { hrs }\end{array}$ & $\begin{array}{c}53 \% \\
48 \mathrm{hrs}\end{array}$ & $\begin{array}{l}51,4 \% \\
48 \mathrm{hrs}\end{array}$ \\
\hline
\end{tabular}

Fuente: elaboración propia.

Este cuadro presenta una aproximación a la dimensión calidad de empleo por medio de tres variables: (se le sugiere numerar las tres variables o marcarlas con letras, por ej. a) formalidad de empleo lo que implica que el trabajador se encuentre registrado y sea sujeto de los derechos sociales respectivos; ingresos se toma como referencia el Salario Mínimo Nacional (smn) hasta el año 2008 el promedio anual dado los reajustes semestrales y desde el 2009 en adelante el valor establecido desde el 1 de enero vigente para cada año analizado y por último, las horas trabajadas semanalmente dado que se considera un categoría de importancia para analizar la calidad de empleo, en el caso

1 Encuesta de Hogares Rurales 2000. MGAP-OPYPA. Dicha encuesta tiene una definición de población rural amplia abarcando a los hogares de las localidades con menos de 5.000 habitantes es decir, 25\% de la población total que no esta integrada a la Encuesta Continua de Hogares (ECH) y sobre la cual se conocía muy poco ya que solo se contaba con los censos nacionales para el análisis de esta población, lo cuales ocurren cada 10 años. (Fuente: elaboración propia). 
uruguayo para estudiar el impacto de la ley de 8 horas para los trabajadores rurales vigente desde diciembre de $2008^{1}$.

Los datos que se presentan son los porcentajes mayoritarios para cada una de las categorías mencionadas, que vale subrayar se aproximan a la dimensión calidad de empleo dado que las diferentes corrientes teóricas agregan otras categorías a la misma, pero no es el objetivo central del trabajo, ni existen las posibilidades metodológicas de recopilar la información necesaria para dar cuenta de tales planteos conceptuales.

Se observa que en materia de formalidad los jóvenes se han empleado en mayor proporción que los adultos en tales condiciones, lo que indica una mejora en este aspecto en relación con la generación anterior y a cambios operados en el escenario de la política pública laboral que han generado no solo controles sino también incentivos económicos para ello. Por otra parte, cabe agregar que en la pasada década se han posicionado empresas multinacionales en la agropecuaria nacional, que han impreso otra dinámica en las relaciones laborales que podrían estar impactando en las generaciones más jóvenes. Pero serían posibles hipótesis a estudiar a posteriori como identificar qué tipos de empleos desarrollan los jóvenes y adultos en condiciones de formalidad o informalidad.

En lo referente al ingreso se aprecia que la evolución del mismo en el último lustro ha tenido movimientos pendulares, pero los mismos han ido favoreciendo a las generaciones más jóvenes dado que en su mayoría perciben salarios por debajo de 1 salario mínimo nacional, con una tendencia decreciente. Situación diferente ocurre entre los adultos. Seguramente, dicha diferenciación se deba a los tipos de ocupaciones que desarrollan, lo que en cuadros anteriores se relacionan en el caso de los jóvenes en actividades del sector secundario y terciario, lo que a su vez tiene asociación con la mayor educación formal de lo mismos comparada con la de sus padres. Cabe señalar que 1 smn en el año 2006 equivalía en promedio a USD 125, en 2007 a USD 137, en 2008 a USD 216, en 2009 a USD 196 y en 2010 a USD 245.

Por último, en materia de horas de trabajo semanal la tendencia general ha sido de aumentar la carga de 48 horas semanales de trabajo en los últimos 5 años, pero con mayor peso de dicha tendencia entre los jóvenes en relación con los adultos, que estaría indicando el tipo de empleo al cual accede el joven comparado con el adulto. Por otro lado, señalaría la capacidad multifuncional y flexible laboral de los jóvenes de articularse a los recientes cambios en el mercado de empleo.

Las variables utilizadas para aproximarse a la calidad del empleo permiten observar una mejora en líneas generales con mayor énfasis entre los jóvenes comparado con los adultos, al acceder los mismos a ocupaciones con mayores grados de formalidad, mejores ingresos y menores cargas de trabajo semanal. La situación 
analizada estaría indicando la existencia de cambios nodales en la configuración de la sociedad rural en estos territorios, dadas las transformaciones económicas, sociales, culturales y políticas acontecidas, lo que implica el desgaste del anterior proceso de configuración social y esta emergencia, recolocando en un papel plurifuncional a dichos territorios.

\section{Conclusiones}

Las transformaciones productivas de los últimos años en el territorio rural uruguayo han generado impactos en la estructura generacional del mercado de empleo, especialmente a lo largo de la segunda parte de la década del 2000, consolidándose los perfiles de los roles a desarrollar en la estructura ocupacional por los jóvenes dado que mayoritariamente conforman la fuerza de trabajo no calificada, pero con una reciente tendencia a comenzar a ocupar empleos de mediana calificación en la rama de los servicios y la industria.

Cuando se observa el comportamiento de las unidades productivas demandantes de fuerza de trabajo juvenil el escenario que se configura es heterogéneo. En el caso de las unidades agrarias se presentan demandando de forma creciente empleo juvenil dado que son un recurso valioso a la hora del empleo, mientras que en las unidades productivas de la industria y de los servicios, especialmente esta última, la demanda estaría siendo específica en cuanto al tipo de formación de los jóvenes para el desempeño de ocupaciones de mediana calificación. Es de señalar, la importancia que estarían presentando las Pymes en los servicios y en donde seguramente los jóvenes cumplen un rol de importancia, pero que las actuales herramientas metodológicas para la recolección de la información se encuentran subregistrando a los mismos, lo que presenta un desafío metodológico para la construcción de estos datos.

Se estaría ante un mercado laboral en líneas generales de baja calificación, que comienza a diferenciar y especializar sus oficios en donde la formación tiene un papel de peso. Por otra parte, en esta línea de análisis las ocupaciones que configuran mejor calidad de empleo (considerando formalidad del empleo, ingresos y horas de trabajo semanal) se encontrarían asociadas a los sectores secundarios y terciarios en su mayoría entre los cuales los jóvenes serían fundamentales para el desarrollo de las mismas dado el nivel educativo formal que poseen. Esto estaría marcando una diferencia generacional en el mercado de trabajo que se encontraría en un proceso de transformaciones y de reestructura, la que impacta plenamente en la población rural no agraria ya que adquiere estratégica importancia para la reproducción de las dinámicas productivas, que han generado las transformaciones productivas mencionadas. Por otra parte, se configuran unidades familiares plurifuncionales que construyen un territorio en donde las interconexiones se multiplican y las antiguas fronteras entre lo urbano y rural quedan difusas y se complejizan (Grammont, 2004).

\begin{tabular}{l|l} 
Para & Romero, J. (julio-diciembre, 2012). Población ocupada juvenil en el mercado de trabajo rural
\end{tabular} 
Por último, tener presente la dimensión generacional estaría posibilitando apreciar la dinámica de los agentes que llevan adelante los procesos socioproductivos en los territorios rurales del Uruguay. Lo que sigue es otra idea en el caso de los jóvenes de qué forma configuran el mercado de ocupaciones agrarias como en las no agrarias lo que ayudaría a un enfoque enriquecedor de dichos procesos sociales. La aplicación de esta dimensión de análisis estaría posibilitando ampliar el foco sobre los fenómenos sociales de la ruralidad latinoamericana, dadas las transformaciones, resistencias y reproducción de las estructuras sociales.

\section{Referencias}

Abramovay, R. (2000). Funções e Medidas da Ruralidade no Desenvolvimento Contemporäneo. Rio de Janeiro. Texto para Discusión (702). Ministério do Planejamento, Orçamento e Gestão - IPEA. Rio de Janeiro.

Brito, F. (2000). "Nova ruralidade" e o conflito entre os aspectos econômicos e sócio-ambientais na criação de unidades de conservação. Rio de Janeiro - Brasil. Ponencia presentada en el X Congreso Mundial de Sociología Rural, 30 de julio al 5 de agosto 2000.

Díez, R. (1989). La estructura ocupacional y los jóvenes en Uruguay. Montevideo: Cepal.

Durston, J. (1998). Juventud y Desarrollo Rural: marco conceptual y contextual. En Serie Politicas Sociales (Chile: CEPAL-Naciones Unidas) N² 28.

Encuesta de Hogares Rurales 2000 (2001). MGAP-OPYPA, Montevideo.

Fuller, A. (1990). From Part Time Farming to Pluriativity: a decade of change in rural Europe. Journal of Rural Studies, London, 6, (4), 361-373.

Gómez, S. (2002) La "Nueva Ruralidad" ¿qué tan nueva? Chile: Universidad Austral de Chile.

Grammont, H. (2004). La nueva ruralidad en América Latina. México, D.F.: Revista Mexicana de Sociología (56). $279-300$.

Kageyama, A. (1998). Pluriatividade e ruralidade: alguns aspectos metodológicos. Pocos de Caldas - Minas Gerais. Ponencia presentada en el XXXVI Congresso Brasileiro de Economia e Sociologia Rural.

Katzman, R. (1989). Pobreza en el Uruguay. Medición y Análisis. FCU, SDCS. Ficha 294.

Lovesio, B. y Viscardi, N. (2003). Los estudios de la mujer y de los jóvenes en la construcción del conocimiento sociológico uruguayo. Revista de Ciencias Sociales, Departamento de Sociología, 16 (21), 79-109.

Piñeiro, D. (1997). Trabajadores rurales y flexibilidad laboral: el caso Uruguay. Ponencia presentada en el Seminario El empleo rural en tiempos de flexibilidad. Buenos Aires: UBA - PIETT.

Rama, G. (1989). La situación de la juventud y los problemas de su inserción en la sociedad. En: Políticas sociales en Uruguay. Educación y juventud. Instituto Nacional del Libro, OPSCEPAL-PNUD. Montevideo, (pp. 100-144).

Para citar este artículo:
Romero, J. (julio-diciembre, 2012). Población ocupada juvenil en el mercado de trabajo rural uruguayo, década 2000. Ánfora, 19 (33), 143-161. Universidad Autónoma de Manizales. ISSN 0121-6538. 
Riella, A.; Romero, J.; Tubío, M. (1999). Modernización agraria y empleo rural: un análisis de sus interrelaciones territoriales entre 1970 1990. Concepción - Chile. Ponencia presentada en el XXII Congreso de la Asociación Latinoamericana de Sociología (ALAS).

Riella, A. y Romero, J. (2003). Nueva ruralidad y empleo no agrícola en el Uruguay. In: Cuadernos del GESA 4 "Territorios y organización social de la agricultura". Argentina. Editorial La Colmena.

Rodríguez, E. (1985). La juventud como movimiento social. Elementos para el estudio del caso uruguayo. En Filgueira, Carlos (comp.) Movimientos Sociales en el Uruguay. Montevideo: CLACSO/CIESU/EBO.

Romero, J. (2008). Dinâmicas das Ocupações Não agrícolas em Territórios de Baixa Densidade Populacional no Uruguai e no Sul da Espanha. Tesis Doctoral, Universidad Federal Rio Grande do Sul.

Schneider, S. (2003). A Pluriatividade na Agricultura Familiar. Porto Alegre - Brasil: Editora UFRGS - Programa de Pós-Graduação em Desenvolvimento Rural.

Solari, A. (1989). La educación preescolar básica y media. En Políticas sociales en Uruguay. Educación y juventud. Instituto Nacional del Libro, Montevideo. OPS-CEPAL-PNUD, (pp. 11-53).

\begin{tabular}{l|l} 
Para & Romero, J. (julio-diciembre, 2012). Población ocupada juvenil en el mercado de trabajo rural
\end{tabular} uruguayo, década 2000. Ánfora, 19 (33), 143-161. Universidad Autónoma de Manizales. ISSN 0121-6538. 